\title{
Highly absorbing gadolinium test device to characterize the performance of neutron imaging detector systems
}

\author{
C. Grünzweig, a) G. Frei, E. Lehmann, G. Kühne, and C. David \\ Paul Scherrer Institut, CH-5232 Villigen PSI, Switzerland
}

(Received 19 February 2007; accepted 12 April 2007; published online 14 May 2007)

\begin{abstract}
We report on the fabrication and application of a novel neutron imaging test device made of gadolinium. It is designed for a real time evaluation of the spatial resolution, resolution direction, and distortions of a neutron imaging detector system. Measurements of the spatial resolution of ${ }^{6} \mathrm{LiF}$ doped $\mathrm{ZnS}$ scintillator screens with different thicknesses and of imaging plates were performed. The obtained results are in good agreement with comparison measurements using the standard knife edge detection method. (C) 2007 American Institute of Physics. [DOI: 10.1063/1.2736892]
\end{abstract}

\section{INTRODUCTION}

Neutron imaging is a nondestructive inspection method used for scientific and industrial applications. Advanced methods such as tomography, ${ }^{1}$ dynamic imaging, ${ }^{2}$ and phasecontrast imaging ${ }^{3}$ take advantage of digital detection systems. Nowadays many applications in neutron radiography and tomography require spatial resolution of better than $50 \mu \mathrm{m}$, which is challenging for existing digital detection systems. ${ }^{4-7}$ In addition, the distortions of a digital detector system should be as small as possible to guarantee the best possible data quality for a tomographic reconstruction. There are two main limitations in the spatial resolution of neutron imaging systems: ${ }^{8}$ (I) the effects in the primary neutron detector (scintillator screen) and (II) the properties of the digital secondary detection system. Methodical and technical improvements concerning the limitation factors with respect to spatial resolution and distortion of the neutron imaging detector system need to be verified by a test object. Such test devices are common for $\mathrm{x}$-ray systems and available on commercial basis. ${ }^{9}$ Since the neutron imaging community is much smaller, there is to our knowledge no two dimensional imaging test device available to test the performance of a neutron detector system. However, the neutron imaging community has a strong interest and already notes a request for such an imaging test tool.

In this article, we report the development of such a two dimensional test device, for which we developed a process for the microstructuring of Gd layers. The performance of various scintillator types in combination with an optimized lens system ${ }^{10}$ was evaluated with this test device at the ICON beam line ${ }^{11}$ at the Paul Scherrer Institut. The lens system was designed for a one-to-one imaging with optimum resolution and efficiency of the scintillators onto a charge-coupled device (CCD) camera. This means that the field of view (FOV) is equal to the chip size in the camera. In addition, the performance of imaging plates in combination with different image readers has been investigated.

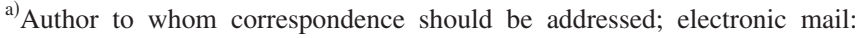
christian.gruenzweig@psi.ch
}

\section{GEOMETRY AND APPLICATION OF THE TEST DEVICE}

A test device suitable for high resolution neutron imaging has to fulfill the following requirements: (I) the contrast in the resulting neutron image should be as high as possible, (II) the feature sizes should cover a wide range down to the expected resolution, and (III) the size of the pattern should fit within the FOV of the detection systems. The developed test device consists of two geometrical patterns, a Siemens star and a square of grid lines. With these two test patterns it is possible to characterize neutron imaging detector systems in terms of the resolution limit, resolution direction, and distortions.

The Siemens star is designed to test the resolution limit in all directions. The Siemens star has a diameter of $20 \mathrm{~mm}$ and is consisting of 128 spokes as shown in Fig. 1(a). The inner spoke period is $40 \mu \mathrm{m}$ ( 25 line pairs $\mathrm{mm}^{-1}$ ) and the outer one is $500 \mu \mathrm{m}$ ( 2 line pairs $\mathrm{mm}^{-1}$ ). Since this test pattern provides the spatial frequencies in all directions the resolution limit can be easily specified from a single neutron image by determining the smallest radius on which the structures can be still resolved. Concentric radial markers at periods of $50,100,200,300,400$, and $500 \mu \mathrm{m}$ were constructed to simplify the reading of the resolution limit.

The second test pattern is a square of grid lines as shown in Fig. 1(b). The size of the square is $24 \times 24 \mathrm{~mm}^{2}$. The distance between two adjacent grid lines is $1 \mathrm{~mm}$. The grid lines have linewidths of 50,100, and $150 \mu \mathrm{m}$. This test pattern is designed to provide a convenient means for measuring distortions, e.g., cushion or a barrel of an imaging detector system, and for a precise measurement of the detector magnification.

Using a lens system designed for one-to-one imaging with optimum resolution of the scintillator light onto a CCD camera the Gd test object holds an important application. To get the maximum amount of light output from the scintillator onto the CCD chip, the diameter of the entrance pupil is chosen maximal. Therefore the focal ratio is small and results in a small depth of focus. If the lens system is first focused with visible light with an object, and later the object 

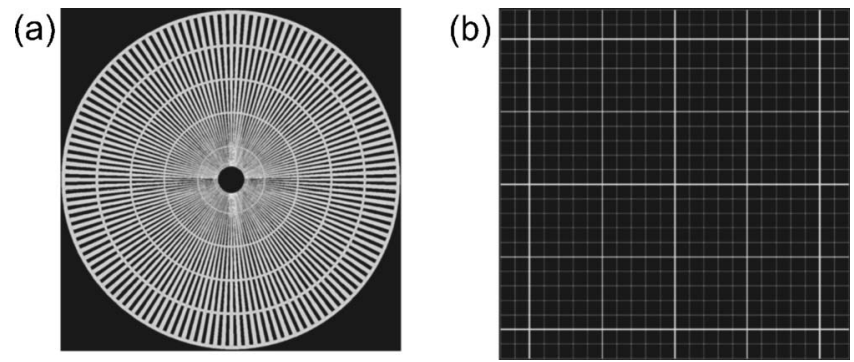

FIG. 1. Geometrical patterns on the test device for the characterization of the detector performance. (a) Geometry of the Siemens star which is ideal for identifying the resolution limit in all directions. (b) Geometry of the square of grid lines which is designed to provide a convenient means for measuring distortions (cushion and barrel).

removed and replaced with the scintillator screen, it is challenging to mount the scintillator within the accuracy of the depth of focus. Unlike with the one dimensional knife edge technique, the focus may be directly determined with scintillation image of the $\mathrm{Gd}$ test device.

\section{MICROFABRICATION OF THE TEST DEVICE}

Test objects for $\mathrm{x}$ rays are usually made of lead or gold structures, which can deliver high absorption contrast for this kind of radiation. They cannot be used for neutrons in the same way, because of the low absorption of these materials. Other materials need to be used in order to obtain a high contrast even in thin layers. Gd has one of the highest thermal neutron capture cross section, e.g., a $6 \mu \mathrm{m}$ thick $\mathrm{Gd}$ layer corresponds to $63 \%$ absorption at a neutron wavelength of $2 \AA$. This provides high contrast for a sufficient neutron image quality.

The test device including the two test patterns described above is fabricated from a $100 \mathrm{~mm}$ diameter quartz wafer. The fabrication process essentially consists of four process steps: (I) In the dc-magnetron sputtering process using argon ions, a three layer system $(\mathrm{Cr} / \mathrm{Gd} / \mathrm{Cr})$ was sputtered onto the quartz substrate. The first layer consists of $25 \mathrm{~nm} \mathrm{Cr}$ to improve adhesion. The second one is a $6 \mu \mathrm{m}$ thick Gd layer to absorb the neutrons. The top $\mathrm{Cr}$ layer with a thickness of $1 \mu \mathrm{m}$ is later needed as an etching mask to pattern the $\mathrm{Gd}$ layer. (II) The transfer of the two test patterns on the layer system was done using optical photolithography. (III) The obtained patterns in the photoresist layer are then transferred by a dry etching process into the $\mathrm{Cr}$ masking layer using chlorine and carbon dioxide as etching gases. (IV) The uncovered regions of the Gd layer are etched away in diluted sulfuric acid (ratio of $1: 100, \mathrm{H}_{2} \mathrm{SO}_{4}: \mathrm{H}_{2} \mathrm{O}$ ). The etching rate is about $100 \mathrm{~nm} \mathrm{~s}^{-1}$.

The resulting structures are shown in Fig. 2. Figure 2(a) shows a light microscopy (LM) image of the inner region of the Siemens star. The smallest spoke period of $40 \mu \mathrm{m}$ is clearly visible. The circle in the image corresponds to the first radial marker indicating the spoke period of $50 \mu \mathrm{m}$. Figure 2(b) shows a LM image of a section of the square of grid lines. The distance between adjacent lines is $1 \mathrm{~mm}$. The three different linewidths of 50, 100, and $150 \mu \mathrm{m}$ are seen.
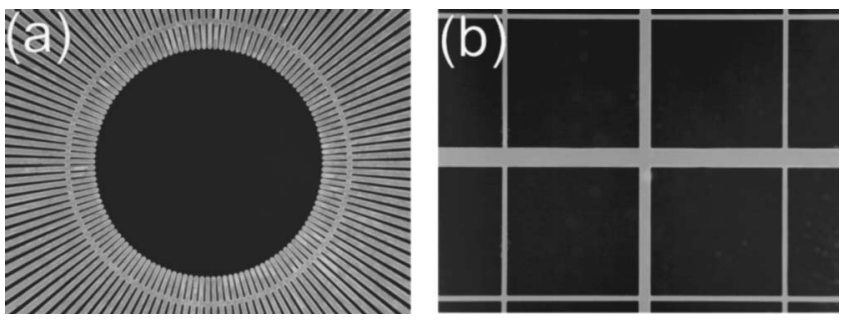

FIG. 2. Light microscopy (LM) images of the fabricated test patterns in the Gd layer. (a) LM image of the inner region of the Siemens star. The Gd lines appear dark. The inner spoke period of $40 \mu \mathrm{m}$ is clearly visible. The radial marker indicates a spoke period of $50 \mu \mathrm{m}$. (b) LM image of a section of the square of grid lines. The three different linewidths of 50,100, and $150 \mu \mathrm{m}$ are visible. The distance between two adjacent lines is $1 \mathrm{~mm}$.

\section{EXPERIMENTAL RESULTS}

We have measured the spatial resolution of a neutron radiography detector system based on ${ }^{6} \mathrm{LiF}$ doped $\mathrm{ZnS}$ scintillator screens with thicknesses of 300, 200, 100, 50, and $25 \mu \mathrm{m}$ using the microfabricated test device. The resolution limit for imaging plates with nominal pixel size provided by the image reader of 50 and $12.5 \mu \mathrm{m}$ is also determined. The performance of the scintillator screens was tested in combination with an one-to-one imaging camera system for which possible emergence of distortions was investigated. The obtained resolution results were cross-checked with measurements using a knife edge technique. The knife edge method evaluates the corresponding modulation transfer function (MTF) which is then used to determine the spatial resolution limit. $^{12,13}$ To determine the MTF of a detector system its edge spread function (ESF) is measured using an opaque object with a straight edge. The ESF is then differentiated to obtain the line spread function from which the MTF is deduced by Fourier transformation. The resolution limit is reached when the MTF drops below 10\%, which corresponds to the so-called cut-off frequency usually given in line pairs $\mathrm{mm}^{-1}$. While this method is the most quantitative way, it has some disadvantages: Within one single knife edge scan one can only determine the resolution in one direction. The knife edge has to be perfectly parallel aligned along a pixel row to get the correct and corresponding ESF. In addition, one has to make some effort concerning the data processing. This is problematic in cases when the resolution needs to be optimized fast, e.g., when searching for the optimum focus setting for the optical system used to project the visible light from a scintillator onto a camera. However, with our test device the information of the whole field of view in all directions is immediately obtained.

As an example, Fig. 3 shows the neutron radiography results obtained with the test device for the 300 and $25 \mu \mathrm{m}$ thick scintillators. The images were taken using the spectrum of the ICON beam line with a maximum flux at around $2 \AA$ and a $L / D$ ratio of 710 , where $L$ and $D$ are the distance and the size of the neutron source. Images (a), (b), and (d) were digital recorded using the one-to-one imaging camera system in combination with the ANDOR DW436 CCD camera which has a chip size of $2048 \times 2048$ pixel and a pixel size of $13.5 \mu \mathrm{m}$ resulting in a FOV of $27 \times 27 \mathrm{~mm}^{2}$. The exposure time for each of the three images was 100 s. Figure 3(a) 

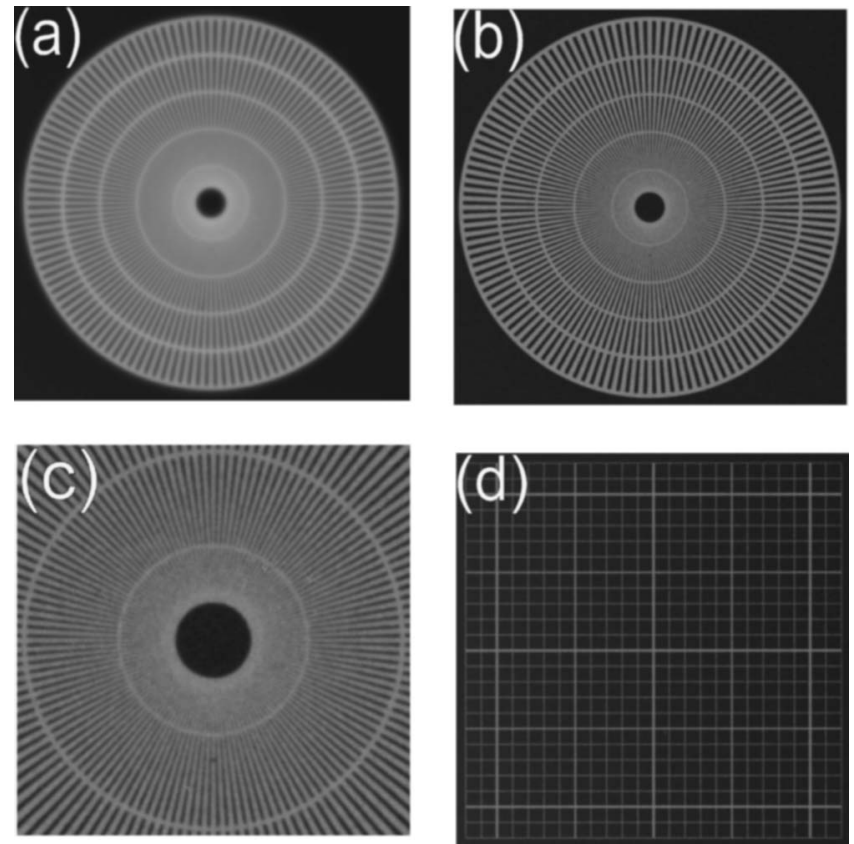

FIG. 3. Neutron radiographs of the Gd test device to characterize the detector performance. All images were taken using a one-to-one imaging camera system: (a) recorded with a $300 \mu \mathrm{m}$ thick ${ }^{6} \mathrm{LiF}$ doped $\mathrm{ZnS}$ scintillator screen (resolution limit around $180 \mu \mathrm{m}$ ), (b) taken with a $25 \mu \mathrm{m}$ thick ${ }^{6} \mathrm{LiF}$ doped $\mathrm{ZnS}$ scintillator screen, (c) is a magnified image of the inner region of image (b) to determine the resolution limit (around $90 \mu \mathrm{m}$ ), and (d) taken with a $25 \mu \mathrm{m}$ thick ${ }^{6} \mathrm{LiF}$ doped $\mathrm{ZnS}$ scintillator. The better resolution of the $25 \mu \mathrm{m}$ thick scintillator and the absence of distortions are obvious.

shows the image of the Siemens star recorded with the $300 \mu \mathrm{m}$ thick scintillator screen. The resolution derived from the image is around $180 \mu \mathrm{m}$ which corresponds to a cut-off frequency of 5.5 line pairs $\mathrm{mm}^{-1}$. The analysis of the MTF measurement resulted in a cut-off frequency of 4 line pairs $\mathrm{mm}^{-1}$ corresponding to a resolution of $250 \mu \mathrm{m}$. The image shown in Fig. 3(b) was taken with the $25 \mu \mathrm{m}$ thick scintillator screen. It is clearly visible that the resolution of this scintillator is better. To determine the resolution limit the inner region of Fig. 3(b) is magnified and shown in Fig. 3(c). The first resolvable white radial marker indicates $100 \mu \mathrm{m}$ spoke period. Hence, the resolution can be determined to about $90 \mu \mathrm{m}$, corresponding to 11.1 line pairs $\mathrm{mm}^{-1}$. The MTF measurement for this screen resulted in a cut-off frequency of 11.6 line pairs $\mathrm{mm}^{-1}$ corresponding to a resolution of $86 \mu \mathrm{m}$.

The results of all resolution measurements are presented in Fig. 4. The comparison measurements for the 25 and $50 \mu \mathrm{m}$ thick ${ }^{6} \mathrm{LiF}$ doped $\mathrm{ZnS}$ scintillator screens and the imaging plates readout with 12.5 and $50 \mu \mathrm{m}$ pixel sizes of the image reader show an excellent agreement. However, the 100, 200, and $300 \mu \mathrm{m}$ thick ${ }^{6} \mathrm{LiF}$ doped $\mathrm{ZnS}$ scintillator screens show some discrepancy. The resolution limit is measured to be better with the Gd test device than for the MTF method, which could be explained by the inaccuracy of aligning the knife edge parallel to a pixel row. The advantage that for a sharp image (i.e., a thin scintillator) the misaligned knife edge could be better seen and therefore better readjusted resulted in a more precise measurement of the MTF as compared to thicker scintillators.

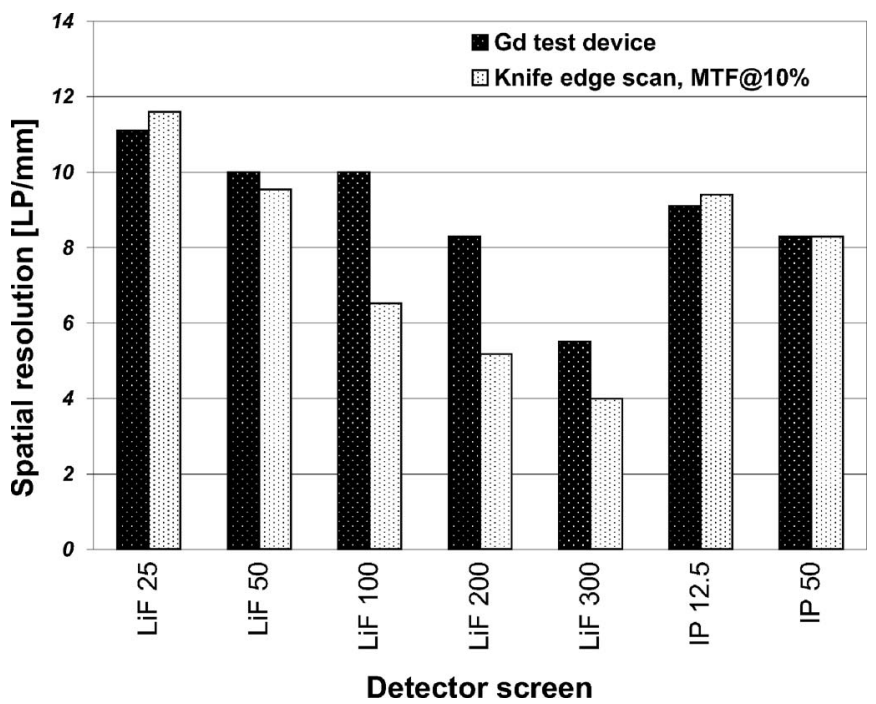

FIG. 4. Comparison of the resolution measurement for different detector screens obtained with the Gd test device and the knife edge method. The results are obtained for ${ }^{6} \mathrm{LiF}$ doped $\mathrm{ZnS}$ scintillator screen with different thicknesses of 25, 50, 100, 200, and $300 \mu \mathrm{m}$ and imaging plate (IP) readout with pixel sizes of 12.5 and $50 \mu \mathrm{m}$.

In addition, it can be seen from Fig. 3(b) that the resolution does not depend on the direction of the spoke structures. Figure 3(d) shows no sign of distortions, since the grid lines are all parallel to each other. These results demonstrate the high performance of the one-to-one imaging camera system.

\section{DISCUSSION}

Based on the presented experimental results we conclude that the developed Gd test device is well suited for characterizing in a quick and semiquantitative way the performance of a neutron imaging detector system. Particularly for determining the spatial resolution of scintillator screens as well as imaging plates, the Gd test device represents an excellent tool. The results obtained with the test device agree well with the resolution measurements done with the knife edge. The distortions of the new detector were measured and could be determined to be negligible. We believe that the Gd test device should be established as a standard device to characterize the equipment on other neutron radiography and tomography beam lines.

\section{ACKNOWLEDGMENTS}

The authors gratefully acknowledge the assistance of E. Deckardt to fabricate the photomask of the test device. They thank I. Johnson, F. Pfeiffer, and O. Bunk for valuable discussions.

${ }^{1}$ P. Vontobel, E. Lehmann, R. Hassanein, and G. Frei, Physica B 385-386, 475 (2006).

${ }^{2}$ R. Hassanein, H. O. Meyer, A. Carminati, M. Estermann, E. Lehmann, and P. Vontobel, J. Phys. D 39, 4284 (2006).

${ }^{3}$ F. Pfeiffer, C. Grünzweig, O. Bunk, G. Frei, E. Lehmann, and C. David, Phys. Rev. Lett. 96, 215505 (2006).

${ }^{4}$ J. S. Brenizer, H. Berger, C. T. Stebbings, and G. T. Gillies, Rev. Sci. Instrum. 68, 3371 (1997).

${ }^{5}$ S. Baechler, N. Kardjilov, M. Dierick, J. Jolie, G. Kühne, E. Lehmann, 
and T. Materna, Nucl. Instrum. Methods Phys. Res. A 491, 481 (2002).

${ }^{6}$ E. Lehmann, P. Vontobel, G. Frei, and C. Brönnimann, Nucl. Instrum. Methods Phys. Res. A 531, 228237 (2004).

${ }^{7}$ N. Kardjilov, S. W. Lee, E. Lehmann, I. C. Lim, C. M. Sim, and P. Vontobel, Nucl. Instrum. Methods Phys. Res. A 542, 100 (2005).

${ }^{8}$ S. Koerner, E. Lehmann, and P. Vontobel, Nucl. Instrum. Methods Phys. Res. A 454, 158 (2000).

${ }^{9}$ XRADIA, x-ray resolution and calibration targets, http://xradia.com/ products/targets.html
${ }^{10}$ E. Lehmann, G. Frei, G. Kühne, and P. Boillat, Nucl. Instrum. Methods Phys. Res. A (in press).

${ }^{11}$ G. Kühne, G. Frei, E. Lehmann, and P. Vontobel, Nucl. Instrum. Methods Phys. Res. A 542, 264270 (2005).

${ }^{12}$ J. Brunner, M. Engelhardt, G. Frei, A. Gildemeister, E. Lehmann, A. Hillenbach, and B. Schillinger, Nucl. Instrum. Methods Phys. Res. A 542, 123128 (2005).

${ }^{13}$ E. Samei, D. A. Reimann, and M. J. Flynn, Med. Phys. 25, 102 (1998). 